\section{IMAGE CHALLENGE}

\section{A 33-year-old man with atypical chest pain}

\section{CLINICAL INTRODUCTION}

A 33-year-old man with no history of coronary artery disease presented to the rapid access cardiology clinic with an episode of atypical anginal chest pain. He had a 15 pack-year history of smoking and a family history of myocardial infarction under the age of 55. Physical examination and exercise ECG testing were unremarkable. On assessment in the cardiology clinic, blood sampling was notable for an elevated high-sensitivity troponin I of $61 \mathrm{ng} / \mathrm{L}$ (99\% upper reference level, $34 \mathrm{ng} / \mathrm{L})$. A coronary CT angiogram was performed (figure 1).

\section{QUESTION}

Which of the following best explains this presentation?

A. Myocarditis

B. Myocardial bridging

C. Kawasaki's disease

D. Atherosclerotic plaque rupture

E. Eosinophilic coronary monoarteritis

For the answer see page 474
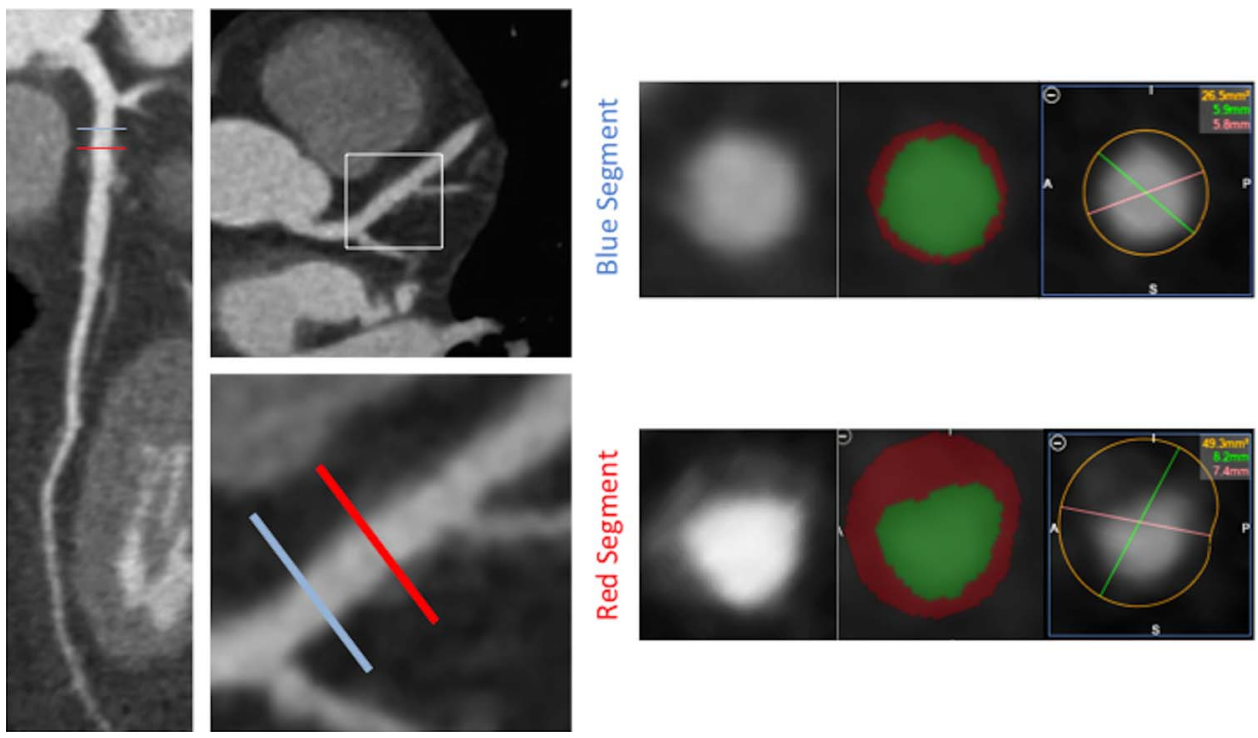

Figure 1 CT multiplanar reformatting of the left anterior descending artery with vessel wall analysis of a proximal (blue) and distal (red) segment. 


\section{A 33-year-old man with atypical chest pain}

For the question see page 455

\section{ANSWER: D}

The correct answer is atherosclerotic plaque rupture. Coronary CT angiography demonstrated a minimally stenotic $(<25 \%)$ noncalcified plaque in the proximal left anterior descending artery (LAD). The vessel wall is thickened with a region of low CT attenuation surrounding the lumen representing a necrotic core of lipid-rich macrophages. ${ }^{1}$ The heterogeneous non-calcified plaque is circumscribed by an outer rim of higher attenuation which is indicative of the napkin-ring sign. Additionally, the plaque has positively remodelled the vessel wall (remodelling index (vessel area in lesion: vessel area in reference segment) of 1.86 , positive remodelling defined as remodelling index $\geq 1.1$ ). In patients with chest pain, the presence of these vulnerable plaque features increases the diagnostic likelihood of acute coronary syndrome. ${ }^{2}$

The presence of coronary risk factors and abnormal coronary artery wall architecture reduces the likelihood of myocarditis. Myocardial bridging frequently occurs in the mid-LAD with plaque development upstream of the bridged segment; however, there is no myocardium surrounding the coronary artery to implicate a 'tunnelled' LAD. Kawasaki's disease often presents in childhood affecting medium-sized arteries and results in luminal dilatation; however, the absence of intraluminal thrombus and coronary artery aneurysm makes this unlikely. ${ }^{3}$ Adventitial infiltration of eosinophils with spontaneous coronary artery dissection of the tunica media is characteristic of eosinophilic coronary monoarteritis and these features are not present in this instance. ${ }^{4}$

The patient was prescribed aspirin and simvastatin. He presented 4 weeks later with an acute thrombotic occlusion of the proximal LAD that required emergency percutaneous coronary intervention (figure 2). Identifying vulnerable plaques may help target effective treatment in individuals with active atherosclerosis to prevent rapid plaque progression and rupture.

\section{Alastair J Moss, ${ }^{1}$ Marc R Dweck, ${ }^{1}$ Andrew D Flapan ${ }^{2}$}

${ }^{1}$ Centre for Cardiovascular Science, University of Edinburgh, Royal Infirmary of Edinburgh, Edinburgh, UK

${ }^{2}$ Edinburgh Heart Centre, Royal Infirmary of Edinburgh, Edinburgh, UK
Correspondence to Dr Alastair J Moss, Centre for Cardiovascular Science, University of Edinburgh, Chancellor's Building, Royal Infirmary of Edinburgh, 51 Little France Crescent, Edinburgh EH16 4TJ, UK; alastairmoss@gmail.com

Contributors AJM and MRD wrote the manuscript and prepared the images. ADF was involved in the clinical care of the patient.

Competing interests None declared.

Provenance and peer review Not commissioned; externally peer reviewed.

\section{(6) OPEN ACCESS}

Open Access This is an Open Access article distributed in accordance with the Creative Commons Attribution Non Commercial (CC BY-NC 4.0) license, which permits others to distribute, remix, adapt, build upon this work non-commercially, and license their derivative works on different terms, provided the original work is properly cited and the use is non-commercial. See: http://creativecommons.org/ licenses/by-nc/4.0/

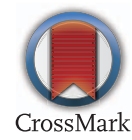

To cite Moss AJ, Dweck MR, Flapan AD. Heart 2016;103:474.

Received 8 July 2016

Revised 17 August 2016

Accepted 24 August 2016

Published Online First 14 September 2016

Heart 2016;103:474. doi:10.1136/heartjnl-2016-310278

\section{REFERENCES}

1 Schlett CL, Maurovich-Horvat P, Ferencik M, et al. Histogram analysis of lipid-core plaques in coronary computed tomographic angiography: ex vivo validation against histology. Invest Radiol 2013;48:646-53.

2 Puchner SB, Liu T, Mayrhofer T, et al. High-risk plaque detected on coronary CT angiography predicts acute coronary syndromes independent of significant stenosis in acute chest pain. Results from the ROMICAT-II trial. J Am Coll Cardiol 2014;64: 684-92.

3 Newburger JW, Takahashi M, Burns JC. Kawasaki Disease. J Am Coll Cardiol 2016:67:1738-49.

4 Robinowitz M, Virmani R, McAllister HA JrU. Spontaneous coronary artery dissection and eosinophilic inflammation: a cause and effect relationship? Am J Med $1982 ; 72: 923-8$.
Figure 2 (A) Coronary

CT angiography of left anterior descending artery (LAD) with non-calcified plaque proximal to first diagonal bifurcation. The arrow points to the culprit plaque in the proximal LAD on the Coronary CT angiogram. (B) Invasive coronary angiogram demonstrated complete occlusion of the proximal LAD. (C) Percutaneous coronary intervention at the site of plaque rupture restored coronary blood flow.
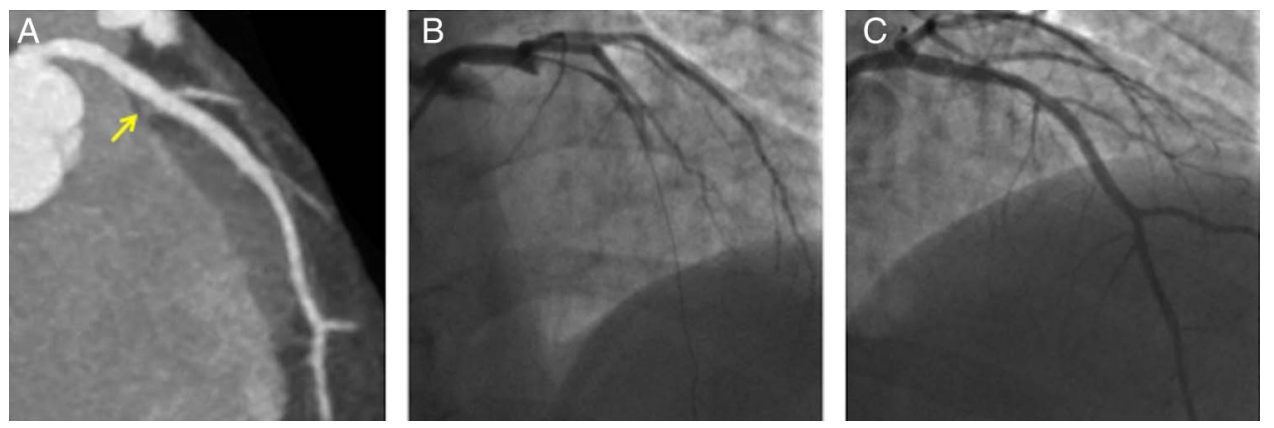